Research Paper

\title{
Prospective matched study on comparison of volumetric-modulated arc therapy and intensity- modulated radiotherapy for nasopharyngeal carcinoma: dosimetry, delivery efficiency and outcomes
}

\author{
Bin-Bin Chen ${ }^{1,2^{*}}$, Shao-Min Huang ${ }^{1 *}$, Wei-Wei Xiao ${ }^{1 *}$, Wen-Zhao Sun ${ }^{1}$, Ming-Zhu Liu ${ }^{1}$, Tai-Xiang Lu ${ }^{1}$, \\ Xiao-Wu Deng', Fei Han ${ }^{1}$ \\ 1. Department of Radiation Oncology, Sun Yat-sen University Cancer Center, State Key Laboratory of Oncology in Southern China, Collaborative Innovation Center for \\ Cancer Medicine, People's Republic of China. \\ 2. Department of Medical Oncology, The Fifth Affiliated Hospital of Sun Yat-sen University, Zhuhai, People's Republic of China. \\ * These authors contributed equally to this work.
}

$\square$ Corresponding author: Fei Han, MD, Department of Radiation Oncology, Sun Yat-sen University Cancer Center, State Key Laboratory of Oncology in Southern China, Collaborative Innovation Center for Cancer Medicine, 651 Dongfeng Road East, Guangzhou 510060, People's Republic of China. Telephone: +86-20-87343543; Fax: +86-20-87343372; E-mail: hanfeisysucc@163.com

(c) Ivyspring International Publisher. This is an open access article distributed under the terms of the Creative Commons Attribution (CC BY-NC) license (https:// creativecommons.org/licenses/by-nc/4.0/). See http://ivyspring.com/terms for full terms and conditions.

Received: 2017.09.16; Accepted: 2018.01.29; Published: 2018.02.28

\begin{abstract}
Background: The purpose of this study is to assess the feasibility of volumetric-modulated arc therapy (VMAT) for nasopharyngeal carcinoma (NPC) patients by comparing the physical dosimetry, delivery efficiency and clinical outcomes with intensity-modulated radiotherapy (IMRT).

Methods: A prospective matched study was performed for patients with newly diagnosed NPC who underwent VMAT or IMRT. The patients in two groups were equally matched in terms of gender, age, tumor stage and chemotherapy. The target coverage, homogeneity index $(\mathrm{HI})$ and conformity index $(\mathrm{Cl})$ of the planning target volume (PTV), organs at risk (OARs) sparing, average treatment time and clinical outcomes were analyzed.

Results: From June 2013 to August 2015, a total of 80 patients were enrolled in this study, with 40 patients in each group. The coverage of PTV was similar for both groups. D2 was observed slight difference only in early stage disease (T1-2) (VMAT vs. IMRT, 7494 \pm 109 cGy vs. 7564 \pm 92 cGy; $\mathrm{p}=0.06$ ). The HI of VMAT group was better than that of IMRT group $(p=0.001)$, whereas $C l$ was slightly worse $(p=0.061)$. The maximum doses received by the brain stem, spinal cord, and optic nerve of VMAT were higher than those of IMRT $(p<0.05)$. But the irradiation volumes in healthy tissue were generally lower for VMAT group, with significant differences in $\mathrm{V} 20, \mathrm{~V} 25$ and V45 ( $<<0.05)$. With regard to the delivery efficiency compared with IMRT $(1160 \pm 204 \mathrm{~s})$, a $69 \%$ reduction in treatment time was achieved by VMAT $(363 \pm 162 \mathrm{~s})$. Both groups had 5 cases of nasopharyngeal residual lesions after radiotherapy. The 2-year estimated local relapse-free survival, regional relapse-free survival and locoregional relapse-free survival, distant metastasis-free survival, disease-free survival and overall survival were similar between two groups, with the corresponding rates of $100 \%, 97.4 \%, 97.4 \%, 90.0 \%, 90.0 \%$ and $92.4 \%$ in VMAT group, and 100\%, 100\%, 100\%, 95.0\%, 95.0\% and 97.5\% in IMRT group, respectively.

Conclusions: Both VMAT and IMRT can meet the clinical requirements for the treatment of NPC. The short-term tumor regression rates and 2-year survival rates with the two techniques are comparable. The faster treatment time benefits of VMAT will enable more patients to receive precision radiotherapy.
\end{abstract}

Key words: nasopharyngeal carcinoma; intensity modulated radiotherapy (IMRT); volumetric modulated arc therapy (VMAT); dosimetry; prognosis

\section{Introduction}

Nasopharyngeal carcinoma (NPC) is distinctly epidemic in Southeast Asia[1], with the age -adjusted incidence rate (per 100,000 people per year) among men of approximately 20-50 in Southern China [2]. 
NPC is a special type of head and neck cancer, most of which in China are undifferentiated non-keratinizing carcinoma [3]. Due to its anatomic characteristics, biological properties and radiosensitivity, radiation therapy has always been the primary treatment modality for nonmetastatic NPC. With continuously improving radiotherapy equipment and technology, intensity modulated radiotherapy (IMRT) is now the current representative accurate radiotherapy technology. Previous studies have reported the great progress of IMRT in the tumor control and quality of life in NPC [4-7].

IMRT can deliver a heterogeneous dose distribution in different target volumes simultaneously, indicating a dose escalation in locality and a dose reduction in adjacent organs at risk [8]. Nevertheless, compared with two-dimensional radiotherapy, the greatest drawback of IMRT is the prolonged delivery time. The prolonged treatments not only decrease efficiency and increase the discomfort and involuntary movements of patients on the couch, which may increase the risk of dose deviation and impair the treatment accuracy, but also will stimulate repair of sublethal damage which is a risk for sparing tumors [9-14].

Volumetric modulated arc therapy (VMAT), a technique first proposed by the Yu's group [15] and developed based on an investigation from Otto [16], can change the dose delivery with various gantry arcs dynamically while the gantry rotates around the patient, due to the capability of continuously modulation of multi-leaf collimator (MLC) positions, dose rate and gantry speed simultaneously [17-19]. Different from IMRT technique with static gantry, VMAT does not require the selection of a finite number of fixed gantry angles and allows greater flexibility because it delivers radiotherapy from all beam orientations. Also, because it can finish the entire treatment in a single $360^{\circ}$ rotation of the gantry, it is potentially more time-saving. VMAT has been widely used by the radiotherapy community and introduced for clinical use in different treatment sites.

Several excellent studies have investigated the differences between VMAT-based treatment plans and IMRT-based plans in NPC at both planning and clinical level [20-25]. However, previous reports on the dosimetric comparisons were primarily based on the same set of CT images from the same patient. When exposing a patient to radiation, treatment can only be implemented using one technique. It is therefore necessary to perform a comparison of the two radiotherapy techniques in patients who had underwent actual clinical radiation exposure. Besides, the clinical benefit from faster treatment is not so clear.

Therefore, we conduct this research, using a prospectively matched method, to investigate the differences in both plan quality and peripheral doses of VMAT and IMRT in NPC patients, leading to a more comprehensive evaluation of the treatment techniques. Furthermore, tumor regression rates and long-term clinical outcomes of the two techniques are compared directly.

\section{Materials and methods}

\section{Clinical characteristics of the patients}

Inclusion criteria: (i) newly diagnosed cases of primary NPC with pathological confirmation; (ii) pathologic type of differentiated non-keratinizing carcinoma or undifferentiated non-keratinizing carcinoma (i.e., WHO type II or type III); (iii) age of 20-80 years old; (iv) male or non-pregnant female; (v) in good general condition, with a Karnofsky Performance Status (KPS) score $\geq 80$; (vi) exhibiting no severe systemic disease or exhibiting complications but expected to complete the radiotherapy; and (vii) no evidence of distant metastasis.

\section{Case grouping}

A prospective patient assignment was performed. Patients receiving treatment with VMAT technique (VMAT group) served as the reference benchmark, and patients receiving treatment with IMRT technique (IMRT group) were selected to match the VMAT group in terms of several variables. The matched patient characteristics included age (the difference $\leq 5$ years), gender, $\mathrm{T}$ stage, $\mathrm{N}$ stage and chemotherapy. The detailed patient assignment procedures are shown in Figure 1. The staging of every patient was performed based on the seventh edition of the American Joint Committee on Cancer (AJCC) in 2009[26].

\section{Radiotherapy}

\section{Position}

All of the patients were in the supine position, with the connecting line of the third cervical vertebrae and the mandibular angled perpendicular to the bed (the head slightly tilted back) and both hands naturally placed on the sides of the body.

\section{Immobilization and CT simulation scan}

The head and upper neck were fixed using a neck and shoulder thermoplastic mask. CT simulation scan was required for all patients, with the scope from the head to the lower edge of the clavicle and a layer thickness of $3 \mathrm{~mm}$. Plain scanning and enhanced scanning were performed. 


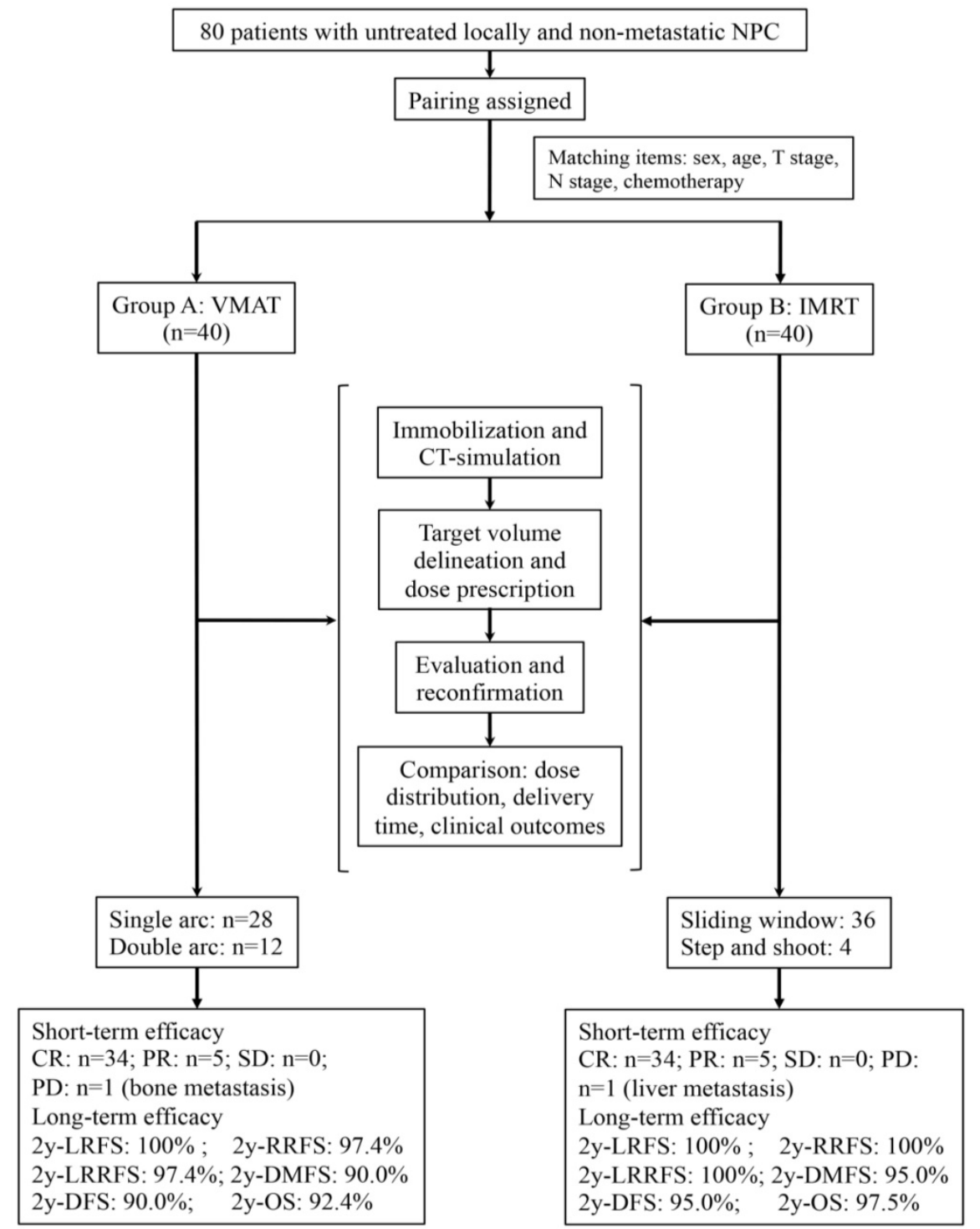

Figure 1. The trial flow chart is shown. NPC indicates nasopharyngeal carcinoma; VMAT, volumetric modulated arc therapy; IMRT, intensity modulated radiation therapy; RT, radiotherapy; CR, complete response; PR, partial response; SD, stable disease; PD, progressive disease; $2 y$, two-year; LRFS, local relapse-free survival; RRFS, regional relapse-free survival; LRRFS, locoregional relapse-free survival; DMFS, distant metastasis-free survival; DFS, disease-free survival; OS, overall survival.

\section{Target volumes and OARs delineation}

The CT images for both groups were imported in the Monaco ${ }^{\circledR}$ (Elekta Medical Systems, Sweden) physician workstation, on which the target area and the area of organs at risk (OARs) were delineated. The gross tumor volumes (GTVs) were divided into nasopharyngeal primary gross tumor volume (GTVnx) and neck metastatic lymph node gross tumor volume (GTVnd). The clinical target volumes (CTVs) were divided into the high-risk area (CTV1) and the low-risk area (CTV2) on the basis of tumor invasion. The various planning target volumes (PTVs) were defined from the respective target volumes extending $3 \mathrm{~mm}$ margins with 3D expansion, corresponding to PGTVnx, PGTVnd, PTV1 and PTV2.
The OARs included the brain stem, spinal cord, temporal lobes, pituitary, optic chiasm, optic nerves, lenses, inner ears, temporomandibular joints, parotid glands, and mandible.

\section{Dose prescription and optimization}

Dose optimization and calculation were performed on the Eclipse treatment planning system (version 10.0 or 11.0, Varian Medical System, USA) or the Monaco treatment planning system (version 3.2, Elekta, Sweden), according to the corresponding radiotherapy machines. Both VMAT and IMRT were generated using a $6 \mathrm{MV}$ X-ray system. Based on the tumor volume and the degree of invasion, a single or double arc design was used in VMAT. A 7- or 9-field design was used for IMRT, with similar optimization 
conditions. The prescribed doses were as follows: 68-72 Gy to the PGTVnx, 64-68 Gy to the PGTVnd, 60 Gy to the PTV1, and 54-56 Gy to the PTV2, in 30-33 fractions. Radiation was delivered once per day, at 5 fractions per week. For all of the plans, the prescribed dose was required to cover at least $95 \%$ of the PTV. Besides, the totally volume that received exceed $110 \%$ of the prescribed dose was restrained less than $20 \%$ inside every PTV but not allowed in any area outside the PTVs. All efforts were made to prevent the dose received by OARs from exceeding the following RTOG0225 and RTOG0615 dose limits: maximum dose (Dmax) <45 Gy for the spinal cord; Dmax <54 Gy for the brain stem, optic nerve and optic chiasm; Dmax <8 Gy for the lens; Dmax <70 Gy for temporomandibular joint; Dmean $<46$ Gy for inner ear; and the percentage of parotid gland volume receiving 30 Gy $(\mathrm{V} 30)<50 \%$.

\section{Evaluations of plans}

The quality of radiotherapy plan was evaluated according to the recommendations of the ICRU83 report. It suggests that using Dmax and the minimum dose (Dmin) of PTVs to evaluate the plan's quality is not so appropriate. Rather, the near maximum dose (D2) and the near minimum dose (D98) are recommended. So, this study used parameters of median dose (D50), D2, D98, the dose received by 95\% of the volume (D95), conformity index (CI) and homogeneity index (HI) to assess the target coverage. The MUs per fraction and average treatment time of the two radiotherapy plans were also considered.

The CI formula[27] is as follows: $\mathrm{CI}=(\mathrm{PTV} r e f / \mathrm{VPTV}) \times(\mathrm{PTV} r e f / V 95 \%)$, where PTVref represents the volume of the PTV receiving $95 \%$ of the prescribed dose, VPTV represents the PTV volume, and V95\% represents the volume covered by the $95 \%$ isodose. The closer the $\mathrm{CI}$ is to 1 , the better the conformity is. The HI formula[28] is as follows: $\mathrm{HI}=(\mathrm{D} 2-\mathrm{D} 98) / \mathrm{D} 50$, where D2, D98 and D50 are the doses received by $2 \%, 98 \%$ and $50 \%$ of the PTV volume. The closer $\mathrm{HI}$ is to 0 , the better the homogeneity is. The parameters for comparing vital OARs were Dmax and Dmean.

In the CT images obtained from the patients, all of the outlined target areas were subtracted from the total volume within the outer contour, and all of the resulting volumes were defined as areas of healthy tissue. The volumes of healthy tissue receiving dose of 5 Gy, 10 Gy, 15 Gy, 20 Gy, 25 Gy, 30 Gy, 35 Gy, 40 Gy and 45Gy were defined as V5, V10, V15, V20, V25, V30, V35, V40 and V45, respectively. The above parameters were analyzed and compared between two plans of techniques.

\section{Chemotherapy}

Overall, 89.5\% (17/19) patients from each group with stage III or IVA-B disease (T3-T4 or N2-N3) received chemotherapy, including concomitant chemotherapy with or without inductive chemotherapy. Those who rejected to receive chemotherapy were due to personal reasons. Inductive chemotherapy consisted of cisplatin with 5-fluorouracil (PF), cisplatin with docetaxel (TP) or a triplet of cisplatin, 5-fluorouracil and docetaxel (TPF) every 3 weeks for two to three cycles. Concomitant chemotherapy was cisplatin weekly $\left(30-40 \mathrm{mg} / \mathrm{m}^{2}\right)$ or on weeks 1, 4 and $7\left(80-100 \mathrm{mg} / \mathrm{m}^{2}\right)$ of radiotherapy.

\section{Evaluation criteria for the efficacy}

The treatment response and short-term efficacy of the primary tumor and the neck lymph nodes were evaluated by physical examination, electronic nasopharyngeal microscopy and Magnetic Resonance Imaging (MRI) 3 months after the end of the radiotherapy. The efficacy evaluation was based on the Response Evaluation Criteria in Solid Tumors guidelines version 1.0 (RECIST v1.0).

\section{Statistical methods}

All of the data were analyzed using SPSS 23.0 software. The t-test for two independent samples was used to compare the differences between two groups. Two-sided tests were performed, and differences with $\mathrm{p}<0.05$ were considered as statistically significance. The short-term efficacy was assessed at the time of three months after the radiotherapy. The follow-up duration was calculated from the first day of therapy to the day of death or last examination. The probabilities of local relapse-free survival (LRFS), regional relapse-free survival (RRFS) and locoregional relapse-free survival (LRRFS), distant metastasis-free survival (DMFS), disease-free survival (DFS), overall survival (OS) were estimated using the Kaplan-Meier method. The authenticity of this article has been validated by uploading the key raw data onto the Research Data Deposit (RDD) public platform (www.researchdata.org.cn), with the approval RDD number as RDDA2017000176.

\section{Results}

\section{General patient characteristics}

From June 2013 to August 2015, a total of 80 patients with NPC who received treatment in the Sun Yat-sen University Cancer Center were included in the study. According to the matching procedures described above, the VMAT and IMRT groups each consisted of 40 cases. The general clinical data of the patients are shown in Table 1. 
Table 1. Clinical Characteristics of patients with VMAT or IMRT

\begin{tabular}{|c|c|c|c|}
\hline Characteristics & VMAT & IMRT & $P$ \\
\hline Sex, no (\%) & & & NS \\
\hline Male & $29(72.5)$ & $29(72.5)$ & \\
\hline Female & $11(27.5)$ & $11(27.5)$ & \\
\hline Age, years & & & 0.725 \\
\hline Median & 50.5 & 51.0 & \\
\hline Range & $22.0-65.0$ & $23.0-67.0$ & \\
\hline T Stage ( $7^{\text {th }}$ AJCC), no. (\%) & & & NS \\
\hline $\mathrm{T} 1$ & $12(30.0)$ & $13(32.5)$ & \\
\hline $\mathrm{T} 2$ & $14(35.0)$ & $13(32.5)$ & \\
\hline $\mathrm{T} 3$ & $9(22.5)$ & $9(22.5)$ & \\
\hline $\mathrm{T} 4$ & $5(12.5)$ & $5(12.5)$ & \\
\hline N Stage (7th AJCC), no. (\%) & & & NS \\
\hline No & $17(42.5)$ & $16(40.0)$ & \\
\hline N1 & $13(32.5)$ & $14(35.0)$ & \\
\hline N2 & $9(22.5)$ & $9(22.5)$ & \\
\hline N3 & $1(2.5)$ & $1(2.5)$ & \\
\hline Clinical stage (7th AJCC), no. (\%) & & & NS \\
\hline I & $8(20.0)$ & $8(20.0)$ & \\
\hline II & $13(32.5)$ & $13(32.5)$ & \\
\hline III & $13(32.5)$ & $13(32.5)$ & \\
\hline IVA & $5(12.5)$ & $5(12.5)$ & \\
\hline IVB & $1(2.5)$ & $1(2.5)$ & \\
\hline \multicolumn{4}{|l|}{ Dose for primary site, $c G y$} \\
\hline Total dose* & 6996 (6789-7029) & $7020(6810-7062)$ & 0.060 \\
\hline Dose/fraction* & $226(212-234)$ & $227(212-234)$ & 0.302 \\
\hline Treatment time, days & & & 0.129 \\
\hline Median & 44 & 43 & \\
\hline Interquartile range & $42-45$ & $42-44$ & \\
\hline Chemotherapy, no. (\%) & & & NS \\
\hline Induction & $1(2.5)$ & $1(2.5)$ & \\
\hline Concurrent & $9(22.5)$ & $9(22.5)$ & \\
\hline Induction + concurrent & $11(27.5)$ & $11(27.5)$ & \\
\hline None & $19(47.5)$ & $19(47.5)$ & \\
\hline
\end{tabular}

Abbreviations: VMAT, volumetric modulated arc therapy; IMRT, intensity modulated radiation therapy; AJCC, American Joint Committee on Cancer; cGy, centigray; NS, nonsignificant.

* Data are presented as median (range).

\section{Comparison of the dose distribution in the PTVs}

All plans met the requirement for the prescribed dose coverage of the target. As shown in Table 2, there were no significant differences in the volumes of PGTVs between the two groups when considering all groups, the locally early stage (T1-2) subgroups and the locally advanced stage (T3-4) subgroups ( $\mathrm{p}=0.745$, 0.199 and 0.201 , respectively). D98, D95 or D50 of the PGTVs were similar, while D2 of the VMAT group was significantly lower than that of the IMRT group $(p=0.025)$. Additionally, subgroup analysis found that the significant difference in D2 was primarily in the early stage disease (T1-2) (VMAT vs. IMRT, 7494 \pm 109 cGy vs. 7564 \pm 92 cGy; $p=0.016$ ).

The comparisons of $\mathrm{HI}$ and $\mathrm{CI}$ between two groups were also shown in Table 2 . The subgroup analysis showed that the difference of $\mathrm{HI}$ was primarily in the early stage disease $(\mathrm{T} 1-2)(\mathrm{p}=0.001)$.
Table 2. Dosimetric comparison of VMAT and IMRT for the PGTVnxs $(\bar{x} \pm s)$

\begin{tabular}{|c|c|c|c|}
\hline Parameters & VMAT & IMRT & $P$ \\
\hline \multicolumn{4}{|l|}{ All $(n=80)$} \\
\hline Volume $\left(\mathrm{cm}^{3}\right)$ & $37.45 \pm 34.72$ & $36.30 \pm 27.87$ & 0.745 \\
\hline D98\# (cGy) & $7151 \pm 103$ & $7158 \pm 89$ & 0.750 \\
\hline D95\& (cGy) & $7189 \pm 102$ & $7197 \pm 90$ & 0.706 \\
\hline D50\& (cGy) & $7335 \pm 99$ & $7365 \pm 97$ & 0.183 \\
\hline $\mathrm{D} 2^{*}(\mathrm{cGy})$ & $7509 \pm 104$ & $7562 \pm 106$ & 0.025 \\
\hline $\mathrm{HI}$ & $0.048 \pm 0.010$ & $0.056 \pm 0.009$ & 0.001 \\
\hline $\mathrm{CI}$ & $0.30 \pm 0.11$ & $0.35 \pm 0.11$ & 0.061 \\
\hline \multicolumn{4}{|l|}{ T1-2 (n=52) } \\
\hline Volume $\left(\mathrm{cm}^{3}\right)$ & $20.65 \pm 15.31$ & $24.62 \pm 10.47$ & 0.199 \\
\hline D98\# (cGy) & $7159 \pm 122$ & $7169 \pm 74$ & 0.705 \\
\hline D95\$ (cGy) & $7191 \pm 122$ & $7208 \pm 77$ & 0.550 \\
\hline D50\& (cGy) & $7330 \pm 118$ & $7368 \pm 83$ & 0.186 \\
\hline $\mathrm{D} 2^{*}(\mathrm{cGy})$ & $7494 \pm 109$ & $7564 \pm 92$ & 0.016 \\
\hline $\mathrm{HI}$ & $0.045 \pm 0.009$ & $0.054 \pm 0.009$ & 0.001 \\
\hline $\mathrm{CI}$ & $0.29 \pm 0.10$ & $0.32 \pm 0.11$ & 0.213 \\
\hline \multicolumn{4}{|l|}{ T3-4 (n=28) } \\
\hline Volume $\left(\mathrm{cm}^{3}\right)$ & $68.64 \pm 39.41$ & $58.00 \pm 36.61$ & 0.201 \\
\hline D98\# (cGy) & $7137 \pm 60$ & $7137 \pm 111$ & 0.997 \\
\hline D95\$ (cGy) & $7186 \pm 48$ & $7178 \pm 111$ & 0.794 \\
\hline D50\& (cGy) & $7346 \pm 51$ & $7360 \pm 123$ & 0.701 \\
\hline $\mathrm{D} 2^{*}(\mathrm{cGy})$ & $7536 \pm 90$ & $7559 \pm 131$ & 0.600 \\
\hline $\mathrm{HI}$ & $0.054 \pm 0.013$ & $0.059 \pm 0.009$ & 0.250 \\
\hline $\mathrm{CI}$ & $0.34 \pm 0.12$ & $0.40 \pm 0.10$ & 0.129 \\
\hline
\end{tabular}

Table 3. Dosimetric comparison of VMAT and IMRT for the organs at risk (OARs) $(\bar{x} \pm s)$

\begin{tabular}{lllll}
\hline OARs & Index & VMAT (cGy) & IMRT (cGy) & $P$ \\
\hline All (n=80) & & & & \\
Brain Stem & Dmax & $5879 \pm 555$ & $5532 \pm 575$ & 0.007 \\
Spinal Cord & Dmax & $4123 \pm 327$ & $3766 \pm 221$ & $<0.001$ \\
Temporal Lobe_L Dmax & $6796 \pm 483$ & $6704 \pm 548$ & 0.428 \\
Temporal Lobe_R & Dmax & $6741 \pm 462$ & $6812 \pm 569$ & 0.541 \\
Pituitary & Dmax & $5872 \pm 810$ & $5546 \pm 1115$ & 0.139 \\
Optic Chiasm & Dmax & $4319 \pm 1682$ & $3925 \pm 1886$ & 0.327 \\
Optic Nerves_L & Dmax & $3550 \pm 1867$ & $2545 \pm 1918$ & 0.020 \\
Optic Nerves_R & Dmax & $3668 \pm 1901$ & $2707 \pm 2031$ & 0.032 \\
Lens_L & Dmax & $527 \pm 247$ & $455 \pm 208$ & 0.160 \\
Lens_R & Dmax & $575 \pm 314$ & $470 \pm 248$ & 0.101 \\
Inner ear_L & Dmean & $4437 \pm 967$ & $4379 \pm 548$ & 0.743 \\
Inner ear_R & Dmean & $4156 \pm 965$ & $4530 \pm 692$ & 0.050 \\
TM Joint_L & Dmean & $4084 \pm 771$ & $3848 \pm 527$ & 0.115 \\
TM Joint_R & Dmean & $4193 \pm 828$ & $4006 \pm 689$ & 0.277 \\
Mandible_L & Dmean & $4039 \pm 495$ & $4050 \pm 405$ & 0.916 \\
Mandible_R & Dmean & $4037 \pm 543$ & $4119 \pm 427$ & 0.458 \\
Parotid_L & Dmean & $3747 \pm 527$ & $3841 \pm 609$ & 0.464 \\
Parotid_R & Dmean & $3880 \pm 648$ & $3861 \pm 384$ & 0.873 \\
T1-2 (n=52) & & & & \\
Brain Stem & Dmax & $5614 \pm 371$ & $5322 \pm 397$ & 0.008 \\
Spinal Cord & Dmax & $4081 \pm 354$ & $3774 \pm 228$ & 0.001 \\
Optic Nerves_L & Dmax & $2738 \pm 1675$ & $2134 \pm 1689$ & 0.201 \\
Optic Nerves_R & Dmax & $2887 \pm 1730$ & $2185 \pm 1685$ & 0.144 \\
T3-4 (n=28) & & & & \\
Brain Stem & Dmax & $6372 \pm 507$ & $5923 \pm 659$ & 0.054 \\
Spinal Cord & Dmax & $4201 \pm 263$ & $3750 \pm 217$ & $<0.001$ \\
Optic Nerves_L & Dmax & $5057 \pm 1138$ & $3309 \pm 2141$ & 0.014 \\
Optic Nerves_R & Dmax & $5117 \pm 1269$ & $3678 \pm 2315$ & 0.055 \\
\hline Abbreviations: VMAT, volumetric modulated arc therapy; IMRT, intensity \\
modulated radiation therapy; OAR, organ at risk; cGy, centigray; T, T stage; L, left; \\
R, right; Dmax, the maximun dose; Dmean, the mean dose. & \\
& & & &
\end{tabular}




\section{Comparison of the dose distribution of sparing OARs and healthy tissue}

The differences in exposure doses of the OARs between the two groups are summarized in Table 3. The Dmax received by the brainstem, spinal cord, and optic nerve in the VMAT group were slightly higher than those in the IMRT group $(p<0.05)$. The irradiation received by most other structures was similar between two groups, including the temporal lobe, pituitary, optic chiasm, lens, and parotid gland.

The subgroup analysis showed that, for the spinal cord, Dmax of VMAT plans was higher significantly, no matter in T1-2 patients or in T3-4 patients $(p<0.05)$. For the brain stem, difference of Dmax was only observed in T1-2 patients.

The irradiation volumes in healthy tissue outside the target areas of the VMAT group were lower than those of the IMRT group, but only V20, V25 and V45 showed statistically significant differences $(p<0.05)$, as shown in Figure 2.

\section{Comparison of the MUs and delivery time}

The number of MU per fraction resulted to be 698.06 \pm 147.07 in VMAT and 1397.01 \pm 329.38 in IMRT $(p=0.001)$. IMRT plans showed values of MUs doubled compared to VMAT plans. The average treatment time for VMAT, as measured manually during treatment delivery, was $363 \pm 162 \mathrm{~s}$ compared to $1160 \pm 204 \mathrm{~s}$ for IMRT ( $\mathrm{p}=0.042)$. VMAT plans resulted in a $69 \%$ reduction in delivery time consumed.

\section{Comparison of the clinical outcomes}

The median follow-up period was 29 months (range, 6-48 months). The results of short-term efficacy showed that the nasopharyngeal lesions in 5 cases in each group had not completely subsided, and distant metastasis occurred in 1 case from each group. All 5 cases of nasopharyngeal residual lesions in the VMAT group had completely subsided by more than 6 months after radiotherapy. The residual lesions in 4 cases in the IMRT group had completely subsided by this time point, while 1 case still exhibited residual lesions in the nasopharyngeal examination one year after radiotherapy. There was no significant difference in the tumor regression rates between the two groups.

The 2-year estimated LRFS, RRFS, LRRFS, DMFS, DFS and OS rates were similar between two groups, with the corresponding rates of $100 \%, 97.4 \%$, $97.4 \%, 90.0 \%, 90.0 \%$ and $92.4 \%$ in VMAT group, and $100 \%, 100 \%, 100 \%, 95.0 \%, 95.0 \%$ and $97.5 \%$ in IMRT group, respectively (Figure 3). Local failure did not occur in both of this two groups until the end of follow-up.

\section{Discussion}

Radiotherapy is the preferred and effective treatment for NPC. The continuous advances in radiotherapy technology have led to significant therapeutic benefits for patients. The results of the clinical application of IMRT showed that it not only significantly improved the local control and long-term survival rates of NPC but also the quality of life of the patients. VMAT technique has been widely accepted in recent years with the biggest advantage of faster treatment time. In addition, it has been confirmed that VMAT has dose distributions comparable to or better than IMRT in the treatment of head and neck tumors and some somatic tumors[29-31].

In previous studies, the comparisons between VMAT and IMRT techniques were based on two sets of plans which were designed on the same target area of the same patient. In actual clinical practice, however, a patient can only receive one plan of

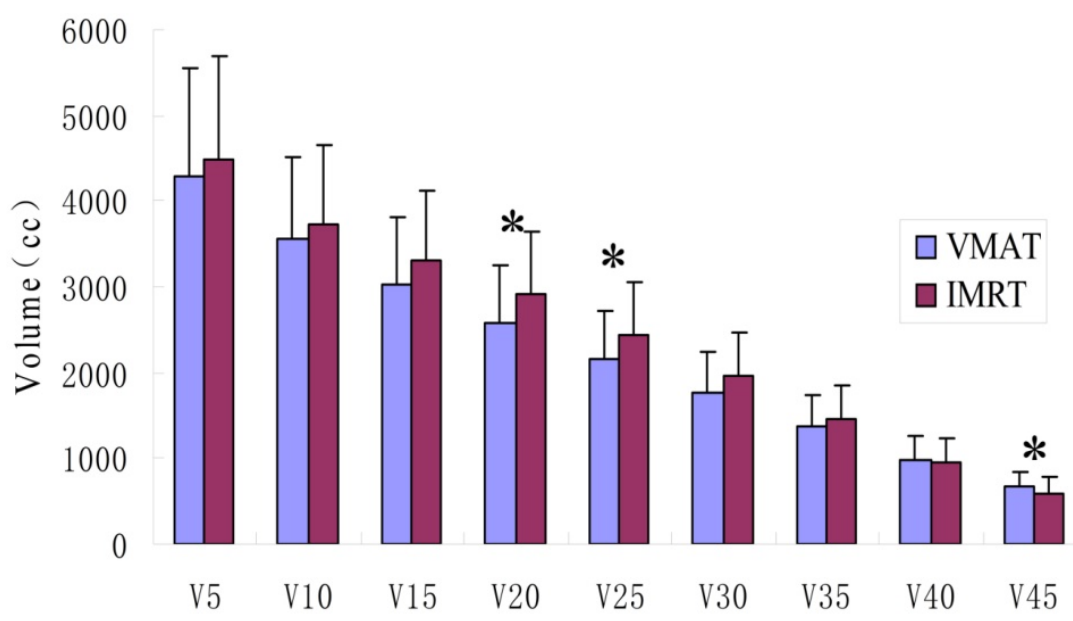

Figure 2. Comparison of the exposure doses and volumes in the healthy tissue. VMAT indicates volumetric modulated arc therapy; IMRT, intensity modulated radiation therapy. V5, V10, V15, V20, V25, V30, V35, V40 and V45 represents the volumes of healthy tissue receiving 5 Gy, 10 Gy, 15 Gy, 20 Gy, 25 Gy, $30 \mathrm{~Gy}, 35 \mathrm{~Gy}, 40 \mathrm{~Gy}$ and $45 \mathrm{~Gy}$, respectively. * represents significant differences $(p<0.05)$. 

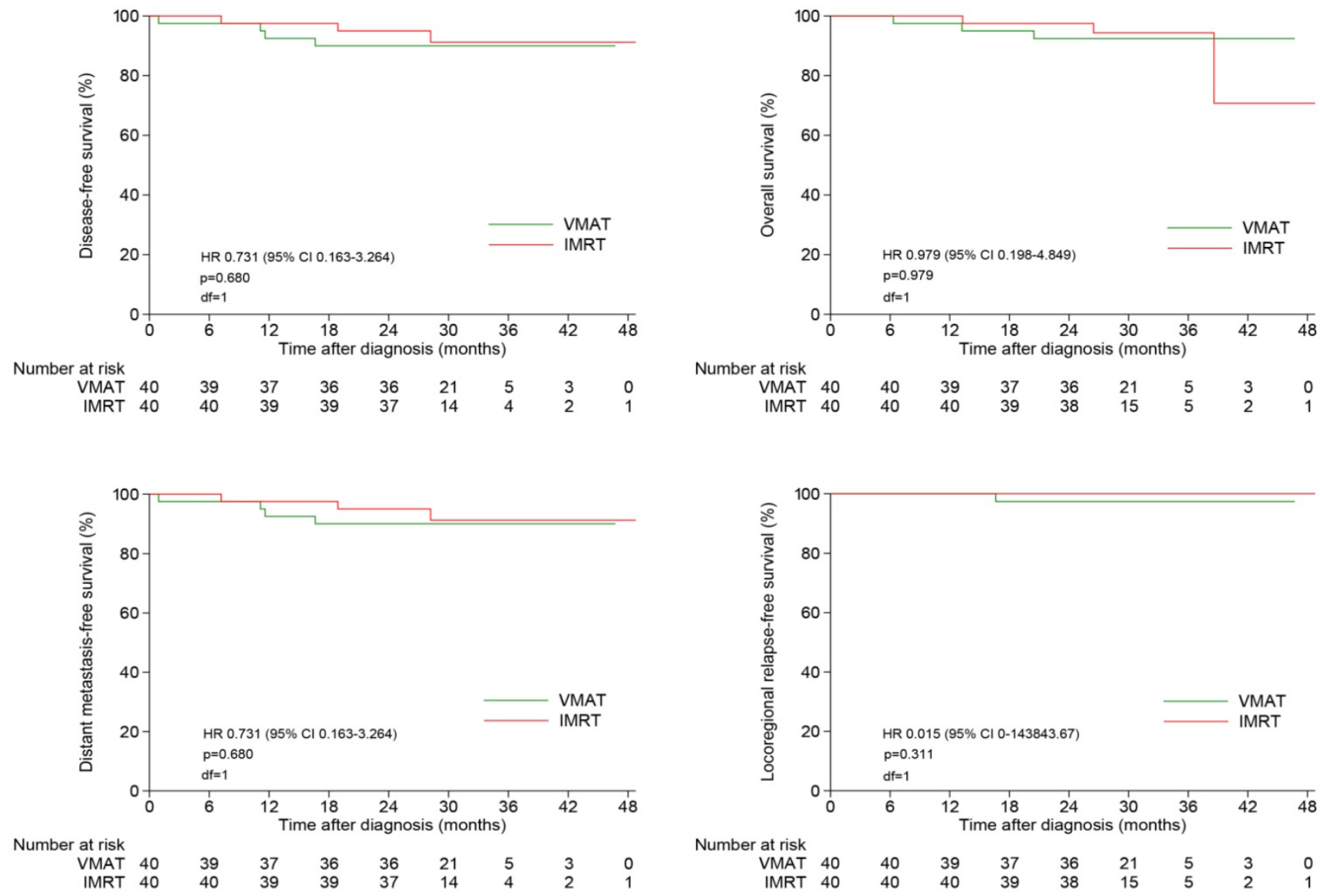

Figure 3. Kaplan-Meier survival curves of NPC patients treated with VMAT and IMRT. NPC indicates nasopharyngeal carcinoma; VMAT, volumetric modulated arc therapy; IMRT, intensity modulated radiation therapy.

treatment. Therefore, prospectively allocating matched patients randomly to either VMAT plan or IMRT plan, as in this study, can better reflect the actual clinical situation. After a scientific and rational allocation, there were no significant differences between the two matched groups in the volume of the gross tumor. Both VMAT and IMRT plans met the clinical requirements of prescribed dose coverage of the PTVs, which is consistent with the findings of Vanetti et al.[32] and Johnston et al.[33].

The comparison of the dose distribution on the target area showed that the VMAT and IMRT plans did not differ a great deal difference overall. VMAT plan owned a significantly lower D2 and a better HI, but a slightly worse $\mathrm{CI}$, the reason of which was possibly related with a more share out of radiation dose by the healthy tissue when gantry rotating (high V40 and V45). Subgroup analysis showed that the difference in homogeneity between the two groups was primarily in the early stage disease. And it seemed that IMRT was more likely to generate an excessive dose which we usually called "hot spot" in the tumor area for the early stage lesion. We considered that it was the intrinsic property of highly conformity of IMRT technique that leading to such steep high-dose gradient at the target edge. Nevertheless, for the locally advanced disease, neither homogeneity nor conformity were seen differences between the two techniques. The reason was that, due to the bulky tumor lesion and the proximity of adjacent vital organs in locally advanced disease, a compromise was made to meet the clinical requirement of radical radiotherapy firstly.

In terms of sparing OARs, IMRT plans protected the brainstem and spinal cord better, which is slightly different from the findings of previous studies. Vanetti et al.[32] reported that the dose of VMAT was $37.7 \%$ lower than that of IMRT in the brainstem and $8.9 \%$ lower in the spinal cord. Similarly, Lee et al.[20] demonstrated that the dose received by brain stem with the VMAT plan was lower than with the IMRT plan in locally advanced NPC. For other OARs, such as the mean dose received by parotid gland, VMAT plan could also make a good constraint. The study of Johnston et al.[33] also provided that VMAT had a greater advantage in protection of parotid gland. The reason for the different results may be because double-arc VMAT technique and step-and-shoot 
IMRT technique were used in the above studies. In contrast, in the present study, single arc VMAT technique was applied in $70 \%(28 / 40)$ of patients in the VMAT group, and dynamic sliding window IMRT technique was applied in $90 \%(36 / 40)$ of patients in the IMRT group. The studies of Guckenberger et al.[34] and Lee et al.[35] have proved that, in terms of dose distribution, double-arc VMAT planning is superior to single-arc planning and that dynamic IMRT is superior to step-and-shoot IMRT.

The exposure volumes of the healthy tissue outside the target area were compared for the two techniques. We found that the exposure volume of healthy tissue in the low-dose area (V5-V35) was smaller for VMAT, especially in the V20-V25 dose range. This finding is consistent with previous reports[20,32]. In cases of long-term survival, this advantage may reduce the risk of secondary tumor induced by radiation.

Our study reported that the clinical outcomes, either for the short-term tumor regression rates or for the 2-year survival rates, were highly similar between VMAT and IMRT plans. This result is consistent with the findings reported by Guo et al.[23]. Guo et al. also revealed that it was the volume of tumor, not the T-staging, associated with advanced disease, poor prognosis, distant metastasis and local recurrence [23]. Reviewed our study, the GTVs between two groups were comparable, which excluded the impact of primary lesion volume on tumor regression. This fact further confirmed the comparable efficacies between the two techniques. Our results also showed that the average MU of the VMAT plan was lower than that of the IMRT plan by approximately $50 \%$, and moreover, VMAT consumed an overwhelming reduction of $69 \%$ in treatment time compared with IMRT. In this respect, VMAT can offer an excellent tumor control more efficiency, which will improve comfort and ensure the stability of the patient's position. Moreover, faster treatment time of each exposure can increase the number of patients who can undergo treatment. Therefore, VMAT radiotherapy equipment can be used in a more efficient manner, and more patients can receive timely treatment.

\section{Conclusion}

In summary, both VMAT and IMRT can meet the clinical requirements for the treatment of NPC. The homogeneity of VMAT in the tumor target area is better, while IMRT can better protect the brain stem and spinal cord in patients with locally early stage disease. The short-term tumor regression rates and 2-year survival rates with the two techniques are comparable. The faster treatment time benefits of
VMAT will enable more patients to receive precision radiotherapy.

\section{Abbreviations}

NPC: nasopharyngeal carcinoma; VMAT: volumetric-modulated arc therapy; IMRT: intensity-modulated radiotherapy; MLC: multi-leaf collimator; AJCC: American Joint Committee on Cancer; OAR: organ at risk; GTV: gross tumor volume; CTV: clinical target volume; PTV: planning target volume; Dmax: maximum dose; Dmin: minimum dose; Dmean: mean dose; D2: the near maximum dose; D98: the near minimum dose; D95: the dose received by $95 \%$ of the volume; CI: conformity index; HI: homogeneity index; MRI: Magnetic Resonance Imaging; RECIST: Response Evaluation Criteria in Solid Tumors guidelines; LRFS: local relapse-free survival; RRFS: regional relapse-free survival; LRRFS: locoregional relapse-free survival; DMFS: distant metastasis-free survival; DFS: disease-free survival; OS: overall survival.

\section{Acknowledgement}

We sincerely thanked professor Ying Guo (Sun Yat-sen University cancer center, State Key Laboratory of Oncology in Southern China, Collaborative Innovation Center for Cancer Medicine, People's Republic of China) for providing support in statistical analysis.

\section{Funding}

This work was supported by funds from the Guangdong Science and Technology Department No. 2012B061700089.

\section{Author contributions}

T.X.L, X.W.D and F.H contributed to study design and conception. B.B.C, S.M.H, W.Z.S and M.Z.L searched the electronic database and extracted data. B.B.C, S.M.H and W.W.X analyzed and interpreted the data. B.B.C and W.W.X contributed to manuscript editing. T.X.L, X.W.D and F.H contributed to quality control and review of the data and manuscript. All authors have read and approved the final version of the submitted manuscript.

\section{Competing Interests}

The authors declare no competing financial interests.

\section{References}

[1] Yu MC, Yuan J-M. Epidemiology of nasopharyngeal carcinoma. Semin Cancer Biol 2002;12:421-9.

[2] Jemal A, Bray F, Center MM, Ferlay J, Ward E, Forman D. Global cancer statistics. CA Cancer J Clin 2011;61:69-90.

[3] Wei WI, Sham JS. Nasopharyngeal carcinoma. The Lancet 2005;365:2041-54. 
[4] Lee N, Xia P, Quivey JM, Sultanem K, Poon I, Akazawa C, et al. Intensity-modulated radiotherapy in the treatment of nasopharyngeal carcinoma: an update of the UCSF experience. Int J Radiat Oncol Biol Phys 2002;53:12-22.

[5] Lee AWM, Sze WM, Au JSK, Leung SF, Leung TW, Chua DTT, et al. Treatment results for nasopharyngeal carcinoma in the modern era: the Hong Kong experience. Int J Radiat Oncol Biol Phys 2005;61:1107-16.

[6] Wolden SL, Chen WC, Pfister DG, Kraus DH, Berry SL, Zelefsky MJ. Intensity-modulated radiation therapy (IMRT) for nasopharynx cancer: update of the Memorial Sloan-Kettering experience. Int J Radiat Oncol Biol Phys 2006;64:57-62.

[7] Xiao W-W, Han F, Lu T-X, Chen C-Y, Huang Y, Zhao C. Treatment outcomes after radiotherapy alone for patients with early-stage nasopharyngeal carcinoma. Int J Radiat Oncol Biol Phys 2009;74:1070-6.

[8] Salz H, Wiezorek T, Scheithauer M, Schwedas M, Beck J, Wendt TG. IMRT with compensators for head-and-neck cancers treatment technique, dosimetric accuracy, and practical experiences. Strahlenther Onkol 2005;181:665-72.

[9] $\mathrm{Mu} \mathrm{X}$, Lofroth P-O, Karlsson M, Zackrisson B. The effect of fraction time in intensity modulated radiotherapy: theoretical and experimental evaluation of an optimisation problem. Radiother Oncol 2003;68:181-7.

[10] Zheng X-K, Chen L-H, Wang W-J, Ye F, Liu J-B, Li Q-S, et al. Impact of prolonged fraction delivery times simulating IMRT on cultured nasopharyngeal carcinoma cell killing. Int J Radiat Oncol Biol Phys 2010;78:1541-7.

[11] Wang JZ, Li XA, D'Souza WD, Stewart RD. Impact of prolonged fraction delivery times on tumor control: a note of caution for intensity-modulated radiation therapy (IMRT). Int J Radiat Oncol Biol Phys 2003;57:543-52.

[12] Fowler JF, Welsh JS, Howard SP. Loss of biological effect in prolonged fraction delivery. Int J Radiat Oncol Biol Phys 2004;59:242-9.

[13] Bewes JM, Suchowerska N, Jackson M, Zhang M, McKenzie DR. The radiobiological effect of intra-fraction dose-rate modulation in intensity modulated radiation therapy (IMRT). Phys Med Biol 2008;53:3567-78.

[14] Jiang L, Xiong X-P, Hu C-S, Ou Z-L, Zhu G-P, Ying H-M. In vitro and in vivo studies on radiobiological effects of prolonged fraction delivery time in A549 cells. J Radiat Res 2013;54:230-4.

[15] Yu CX. Intensity-modulated arc therapy with dynamic multileaf collimation: an alternative to tomotherapy. Phys Med Biol 1999;40:1435-49.

[16] Otto K. Volumetric modulated arc therapy: IMRT in a single gantry arc. Med Phys 2008;35:310-7.

[17] Bortfeld T, Webb S. Single-Arc IMRT? Phys Med Biol 2009;54:N9-20.

[18] Bedford JL, Warrington AP. Commissioning of volumetric modulated arc therapy (VMAT). Int J Radiat Oncol Biol Phys 2009;73:537-45.

[19] Yu CX, Tang G. Intensity-modulated arc therapy: principles, technologies and clinical implementation. Phys Med Biol 2011;56:R31-54.

[20] Lee T-F, Chao P-J, Ting H-M, Lo S-H, Wang Y-W, Tuan C-C, et al. Comparative analysis of SmartArc-based dual arc volumetric-modulated arc radiotherapy (VMAT) versus intensity-modulated radiotherapy (IMRT) for nasopharyngeal carcinoma. J Appl Clin Med Phys 2011;12:3587.

[21] Sun Y, Guo R, Yin W-J, Tang L-L, Yu X-L, Chen M, et al. Which T category of nasopharyngeal carcinoma may benefit most from volumetric modulated arc therapy compared with step and shoot intensity modulated radiation therapy. PLoS ONE 2013;8:e75304.

[22] Ning Z-H, Mu J-M, Jin J-X, Li X-D, Li Q-L, Gu W-D, et al. Single arc volumetric-modulated arc therapy is sufficient for nasopharyngeal carcinoma: a dosimetric comparison with dual arc VMAT and dynamic MLC and step-and-shoot intensity-modulated radiotherapy. Radiat Oncol 2013;8:237.

[23] Guo R, Tang L-L, Mao Y-P, Zhou G-Q, Qi Z-Y, Liu L-Z, et al. Clinical Outcomes of Volume-Modulated Arc Therapy in 205 Patients with Nasopharyngeal Carcinoma: An Analysis of Survival and Treatment Toxicities. PLoS ONE 2015;10:e0129679.

[24] Gao J, Qian T-L, Tao C-Z, Zhang Y-H, Zhou Y, Yang J, et al. SmartArc-based volumetric modulated arc therapy can improve the middle ear, vestibule and cochlea sparing for locoregionally advanced nasopharyngeal carcinoma: a dosimetric comparison with step-and-shoot intensity-modulated radiotherapy. Br J Radiol 2015;88:20150052.

[25] Radhakrishnan S, Chandrasekaran A, Sarma Y, Balakrishnan S, Nandigam J. Dosimetric Comparison between Single and Dual Arc-Volumetric Modulated Arc Radiotherapy and Intensity Modulated Radiotherapy for Nasopharyngeal Carcinoma Using a Simultaneous Integrated Boost Technique. Asian Pac J Cancer Prev 2017;18:1395-402.

[26] Edge SB, Compton CC. The American Joint Committee on Cancer: the 7th edition of the AJCC cancer staging manual and the future of TNM. Ann Surg Oncol 2010;17:1471-4.

[27] Feuvret L, Noël G, Mazeron J-J, Bey P. Conformity index: a review. Int J Radiat Oncol Biol Phys 2006;64:333-42.

[28] Mrozowska M, Kukołowicz P. Relationships between various indices of doses distribution homogeneity. Reports of Practical Oncology and Radiotherapy 2015;20:278-83.

[29] Verbakel WFAR, Cuijpers JP, Hoffmans D, Bieker M, Slotman BJ, Senan S. Volumetric intensity-modulated arc therapy vs. conventional IMRT in head-and-neck cancer: a comparative planning and dosimetric study. Int J Radiat Oncol Biol Phys 2009;74:252-9.

[30] Clivio A, Fogliata A, Franzetti-Pellanda A, Nicolini G, Vanetti E, Wyttenbach $\mathrm{R}$, et al. Volumetric-modulated arc radiotherapy for carcinomas of the anal canal: A treatment planning comparison with fixed field IMRT. Radiother Oncol 2009;92:118-24.

[31] Shaffer R, Morris WJ, Moiseenko V, Welsh M, Crumley C, Nakano S, et al. Volumetric modulated Arc therapy and conventional intensity-modulated radiotherapy for simultaneous maximal intraprostatic boost: a planning comparison study. Clin Oncol (R Coll Radiol) 2009;21:401-7.

[32] Vanetti E, Clivio A, Nicolini G, Fogliata A, Ghosh-Laskar S, Agarwal JP, et al. Volumetric modulated arc radiotherapy for carcinomas of the oro-pharynx, hypo-pharynx and larynx: a treatment planning comparison with fixed field IMRT. Radiother Oncol 2009;92:111-7.

[33] Johnston M, Clifford S, Bromley R, Back M, Oliver L, Eade T. Volumetric-modulated arc therapy in head and neck radiotherapy: a planning comparison using simultaneous integrated boost for nasopharynx and oropharynx carcinoma. Clin Oncol (R Coll Radiol) 2011;23:503-11.

[34] Guckenberger M, Richter A, Krieger T, Wilbert J, Baier K, Flentje M. Is a single arc sufficient in volumetric-modulated arc therapy (VMAT) for complex-shaped target volumes? Radiother Oncol 2009;93:259-65.

[35] Lee TF, Ting HM, Chao PJ, Fang FM. Dual arc volumetric-modulated arc radiotherapy (VMAT) of nasopharyngeal carcinomas: a simultaneous integrated boost treatment plan comparison with intensity-modulated radiotherapies and single arc VMAT. Clin Oncol ( $\mathrm{R}$ Coll Radiol) 2012;24:196-207. 